www.nature.com/clinicalpractice/neph

elevated levels of parathyroid hormone plus end-stage renal disease.

Using FAST-GATED HELICAL CT, coronary artery calcification (CAC) and carotid calcification were measured, preoperatively and postoperatively, in dialysis patients undergoing subtotal parathyroidectomy $(n=10)$. Randomly selected dialysis patients who did not undergo parathyroidectomy $(n=10)$ were used as a reference.

There was a significant decline or stabilization of CAC in the three parathyroidectomy patients with the highest baseline CAC scores. Changes in CAC score/year in these patients were $-581,-1,180$ and +28.6 . Only one individual with severe calcification at baseline showed a marked increase after gland excision. By contrast, patients in the reference group with very high baseline calcification scores demonstrated large increases in CAC and carotid calcification over time.

Previous studies have hinted at a link between parathyroidectomy and cardiovascular calcification. In contrast to the results reported here, some studies indicate that the procedure actually exacerbates deposition of calcium salts. Because of recent advances in techniques for quantifying cardiovascular calcification, however, the conclusions of the present study could be the most accurate to date.

Rachael Williams

Original article Bleyer AJ et al. (2005) Changes in cardiovascular calcification after parathyroidectomy in patients with ESRD. Am J Kidney Dis 46: 464-469

\section{RAS gene mutations linked to autosomal recessive renal tubular dysgenesis}

According to a recent study, genetic defects in the renin-angiotensin system (RAS) are linked to, and could be the fundamental cause of, autosomal recessive renal tubular dysgenesis (RTD), a severe fetal disorder of kidney development associated with anuria, hypotension and perinatal death. This important discovery might eventually aid early prenatal diagnosis and present genetic counseling opportunities for affected families.

Gribouval et al. analyzed the kidneys of 16 deceased fetuses and neonates with RTD, from nine families (five with a common bloodline). There was an absence of renin RNA or protein expression in three families and enhanced expression in six. All kidneys had limited differentiation of proximal tubules.

Linkage analyses and mutation screening revealed abnormalities in the genes encoding renin, angiotensinogen, angiotensin-converting enzyme or angiotensin II receptor type I. Interestingly, in one family, no mutations of RAS genes were detected, indicating that other genetic factors might also be involved in RTD.

All components of RAS are vital for the vasoconstrictor action of angiotensin II. Loss of vasocontriction would lead to chronic low perfusion pressure in the fetal kidney, and the authors propose that this is the mechanism connecting RAS gene mutations to RTD.

Rachael Williams

Original article Gribouval O et al. (2005) Mutations in genes in the renin-angiotensin system are associated with autosomal recessive renal tubular dysgenesis. Nat Genet 37: 964-968

\section{Equation improves dialysis management of methanol and ethylene glycol poisoning}

A quality assurance study has validated a formula that forecasts hemodialysis time for patients with methanol or ethylene glycol poisoning. The required variables are initial toxin level, dialyzer specifications and patient weight, height, sex and age.

Originally devised by researchers at Dalhousie University's Division of Nephrology in Canada, the equation was tested in 13 patients aged 19-54 years (12 male) with methanol or ethylene glycol poisoning dialyzed using the Fresenius ${ }^{\circledR}$ F8 dialyzer (Fresenius AG, Bad Homburg, Germany). To guarantee that the desired serum toxin levels were reached $(\leq 8 \mathrm{mM}$ methanol or its metabolite formic acid, and $\leq 6 \mathrm{mM}$ ethylene glycol or its metabolite glycolic acid), therapy was continued until the formula predicted blood toxin concentration to be $\leq 5 \mathrm{mM}$. As an extra precaution, toxin levels were measured $2 \mathrm{~h}$ before this point to ensure that dialysis could be safely discontinued.

No significant differences between actual dialysis times (mean $8.4 \pm 3.2 \mathrm{~h}$ ) and the corresponding predicted values (mean $8.7 \pm 3.4 \mathrm{~h}$ ) were found. No rebound toxin increases

\section{GLOSSARY}

FAST-GATED HELICAL CT

The integration of electrocardiogram gating with spiral or multidetector CT scanners with subsecond temporal resolution, such that the $\mathrm{CT}$ images are acquired in respect to the systolic and diastolic phases of the cardiac cycle 